Pacific Journal of Mathematic 


\section{VERTICALLY COUNTABLE SPHERES AND THEIR WILD SETS}

\section{D. LOVELAND}

A 2-sphere $S$ in $E^{3}$ is said to have vertical order $n$ if the intersection of each vertical line with $S$ contains no more than $n$ points. It is shown that $S \cup \operatorname{Int} S$ is a 3-cell that is locally tame from Ext $S$ modulo a 0 -dimensional set if $S$ has vertical order 5 . A subset $X$ of $E^{3}$ is said to have countable (finite) vertical order if the intersection of $X$ with each vertical line consists of countably (finitely) many points. A 2sphere in $E^{s}$ with countable vertical order can have a wild set of dimension no larger than one.

For each 2-sphere $S$ in $E^{3}$ there is a homeomorphism $h: E^{3} \rightarrow E^{3}$ such that each vertical line intersecting $h(S)$ does so in a 0 -dimensional set [2, Theorem 10.1]; thus the condition that a 2-sphere be "vertically 0-dimensional" imposes no restriction on the wildness of the 2-sphere. A study of vertically finite 2-spheres (spheres with finite vertical order) was begun in [10] where it was proven that a 2 -sphere in $E^{3}$ having vertical order 3 is tame. Even though there are wild 2-spheres having vertical order 4 , it is known that $S \cup \operatorname{Int} S$ is a 3-cell if $S$ has vertical order 5 [11]. We extend this result to show that the set $W(S)$ of points where the 2-sphere $S$ fails to be locally tame must be 0-dimensional if $S$ has vertical order 5 . An example is given at the end of the paper to show that 5 is the largest integer for which this result is true. We also show that the wildness of a vertically countable sphere is limited to a 1-dimensional set.

In the remainder of the paper we use $\pi: E^{3} \rightarrow E^{2}$ to denote the vertical projection of $E^{3}$ onto the horizontal plane $E^{2}$. For convenience, we always assume that $E^{2}$ is located vertically below the sphere or cube under investigation. We use $L(x)$ to denote the vertical line containing the point $x$.

A vertical line $L$ is said to pierce a subdisk $D$ of a 2 -sphere $S$ if there is an interval $I$ in $L$ such that $I \cap S$ is a point $p \in D$ and $I$ intersects both Int $S$ and Ext $S$. We say that $L$ links the boundary $\operatorname{Bd} D$ of a disk $D$ if $L$ intersects every disk bounded by $\operatorname{Bd} D$.

2. Spheres having countable vertical order.

THeOREM 2.1. If $S$ is a 2-sphere in $E^{3}$ having countable vertical order, then $W(S)$ contains no open subset of $S$.

Proof. Suppose that $W(S)$ contains a disk $D$ in $S$. We shall 
produce a contradiction by exhibiting a vertical line $L$ whose intersection with $D$ contains a Cantor set.

Assertion A. If $D^{\prime}$ is a subdisk of $D$, then there is an open subset $U$ of $E^{3}$ such that $\pi(U) \subset \pi\left(D^{\prime}\right)$.

To prove Assertion A it suffices to show that $\pi\left(D^{\prime}\right)$ is not onedimensional. This follows from [9, Theorem VI.7, p. 91] since the map $\pi \mid D^{\prime}: D^{\prime} \rightarrow \pi(D)$ is closed.

Assertion B. If $D^{\prime}$ is a subdisk of $D$ and $U$ is an open subset of $E^{3}$ such that $\pi(U) \subset \pi\left(D^{\prime}\right)$, then there exist disjoint disks $D_{1}$ and $D_{2}$ in $D^{\prime}$ and an open subset $N$ of $U$ such that each vertical line through cl $(N)$ intersects both $D_{1}$ and $D_{2}$.

In order to select the disks $D_{i}$ in Assertion B we first show the existence of a vertical line $L$ containing two points $r$ and $t$ in $D^{\prime}$ and containing two sequences $\left\{u_{i}\right\}$ and $\left\{l_{i}\right\}$ of points such that

(1) $\left\{u_{i}\right\}$ converges to $r$ from above,

(2) $\left\{l_{i}\right\}$ converges to $r$ from below,

(3) there is a component $V_{1}$ of $E^{3}-S$ containing every $u_{i}$, and

(4) $E^{3}-\left(S \cup V_{1}\right)=V_{2}$ contains every $l_{i}$.

Notice that some vertical line $L^{\prime}$ intersects $D^{\prime}$ in more than two points [7, Theorem 2.3], so we may choose two points $r^{\prime}$ and $t^{\prime}$ in $L^{\prime} \cap D^{\prime}$. $L$ et $B$ be an open ball centered at $r^{\prime}$ such that $B \cap S \subset D^{\prime}$. If $r^{\prime}$ does not satisfy the four conditions above relative to $L^{\prime}$, it must be because some interval $I$ in $L^{\prime} \cap B$ has $r^{\prime}$ as its midpoint and lies, except for $r^{\prime}$, in a single component, say $V_{1}$, of $E^{3}-S$. Let $B_{1}$ and $B_{2}$ be disjoint round open balls of equal radius centered at points of $L^{\prime}$ above and below $r^{\prime}$, respectively such that $B_{1} \cup B_{2} \subset V_{1} \cap B$. Now close to $r^{\prime}$ and vertically between $B_{1}$ and $B_{2}$, there must exist a point $e$ of $V_{2}$. Then $L=L(e)$ intersects $V_{2}$ between its two intersections with $V_{1} \cap\left(B_{1} \cup B_{2}\right)$, so $L$ intersects $D^{\prime}$ at least twice. Let $r$ be the lowest point of the component of $L \cap\left(S \cup V_{1}\right)$ containing $L \cap B_{1}$, and choose $t$ to be some other point of $L \cap S$. Since $S$ has countable vertical order it is clear that $r$ is a limit point of $L \cap V_{1}$ from above and of $L \cap V_{2}$ from below. Thus conditions (1), (2), (3), and (4) are satisfied.

Choose a disk $D_{1}$ in $D^{\prime}$ such that $r \in \operatorname{Int} D_{1}$ and $t \notin D_{1}$. We claim that there is an open set $U_{1}$ containing $r$ such that every vertical line through $U_{1}$ intersects $D_{1}$. Suppose there is no such open set, and for each $i$ let $E_{i}$ be a horizontal disk centered at $l_{i}$ and lying in $V_{2}$. There must be a sequence $\left\{x_{i}\right\}$ such that $x_{i} \in E_{i}$, for each $i$, no $L\left(x_{i}\right)$ intersects $D_{1}$, and $\left\{L\left(x_{i}\right)\right\}$ converges to $L(r)$. For erch $i$ let $y_{i}$ be the first point of $S$ above $x_{i}$ on $L\left(x_{i}\right)$ (such a point will exist for suf- 
ficiently large integers $i$ since $u_{i}$ and $l_{i}$ are different components of $\left.E^{3}-S\right)$, and let $I_{i}$ be the vertical interval $\left[x_{i}, y_{i}\right]$ in $S \cup V_{2}$. Since some subsequence of $\left\{y_{i}\right\}$ converges, we assume for notational convenience that $\left\{y_{i}\right\}$ converges to a point $y$. Of course $y \in L(r) \cap S$. It is clear that $y$ is not above $r$ on $L(r)$ because $\{r, y\} \subset \lim \inf I_{i} \subset S \cup V_{2}$ whereas $\left\{u_{i}\right\} \rightarrow r$ and $u_{i} \in V_{1}$. Nor is $y$ below $r$ on $L(r)$ because $\left\{l_{i}\right\} \rightarrow r$, $\left\{l_{i}, x_{i}\right\} \subset E_{i}$, and $x_{i}$ lies vertically below $y_{i}$. Thus $\left\{y_{i}\right\}$ converges to $r$, and we have the contradiction that most of the $y_{i}^{\prime} \mathrm{s}$ must belong to $D^{\prime}$ while $L\left(y_{i}\right) \cap D^{\prime}$ was supposed to be empty. The existence of $U_{1}$ is established.

Now choose a disk $D_{2}$ such that $D_{1} \cap D_{2}=\varnothing, t \in \operatorname{Int} D_{2}, D_{2} \subset D^{\prime}$, and $\pi\left(D_{2}\right) \subset \pi\left(U_{1}\right)$. From Assertion A there is an open set $U_{2}$ such that every vertical line through $U_{2}$ intersects $D_{2}$. Such a line will also intersect $U_{1}$ and hence $D_{1}$. Choose $N$ to be any open subset of $U$ such that $\pi\left(\operatorname{cl}(N) \subset \pi\left(U_{1}\right) \cap \pi\left(U_{2}\right)\right)$.

Now that the two assertions have been proven it might be clear how to proceed inductively to produce a vertical line containing uncountably many points of $S$; nevertheless, we give a brief outline. From Assertion A there is an open set $U$ such that every vertical line through $U$ intersects $D$. Now we apply Assertion B to obtain an open set $U_{1}$, whose closure lies in $U$, and two disjoint disks $D_{1}$ and $D_{2}$ in $D$ such that every vertical line through cl $\left(U_{1}\right)$ intersects both $D_{1}$ and $D_{2}$. This ends the first step in the construction. Assertion $\mathrm{B}$ can now be applied to $D_{1}$ to obtain two disjoint disks $D_{11}$ and $D_{12}$ in $D_{1}$ and an open set $N_{1}$ such that vertical lines through cl $\left(N_{1}\right)$ intersect both $D_{11}$ and $D_{12}$. Now $B$ is applied to $D_{2}$ and $N_{1}$ so that at the completion of step 2 we have an open set $U_{2}$ whose closure lies in $U_{1}$ and four disjoint disks $D_{11}, D_{12}, D_{21}$, and $D_{22}$ in $D$ where each vertical line through $\mathrm{cl}\left(U_{2}\right)$ intersects each of the four disks. When the construction is finished it is clear that a vertical line through $\bigcap_{1}^{\infty} \mathrm{cl}\left(U_{i}\right)$ will intersect each of the $2^{n}$ disks at the $n$th step. Thus such a line contains an uncountable set of points of $S$. This contradiction establishes the theorem.

COROLLARY 2.2. If $S$ is a 2-sphere in $E^{3}$ having countable vertical order, then $S$ is locally tame modulo a 1-dimensional subset.

3. Spheres of vertical order order 5. The following four lemmas are used to establish the main result (Theorem 3.5).

Lemma 3.1. If $S$ has vertical 5 , then $S$ is locally tame at each point of $S$ that is vertically above or below a point of Int $S$; that is, $\pi(\operatorname{Int} S) \cap \pi(W(S))=\varnothing$.

Proof. Let $p$ be a point of $S$ such that $L(p) \cap \operatorname{Int} S \neq \varnothing$. Thus 
$L(p)$ must link the boundaries of each of two disjoint disks $D_{1}$ and $D_{2}$ in $S$. Let $B$ be a ball lying in Int $S$ such that each vertical line through $B$ links both $\mathrm{Bd} D_{1}$ and $\mathrm{Bd} D_{2}$. If $p \notin D_{1} \cup D_{2}$, then there is a disk $D_{3}$ in $S$ such that $p \in \operatorname{Int} D_{3}, D_{3} \cap\left(D_{1} \cup D_{2}\right)=\varnothing$, and $\pi\left(D_{3}\right) \subset \pi(B)$. Then each vertical line intersecting $D_{3}$ also intersects both $D_{1}$ and $D_{2}$. Since $D$ has vertical order 5 it is clear that $D_{3}$ has vertical order 3 . Thus $D$ is locally tame at $p[7$, Theorem 2.3] and so is $S$.

We may now assume that $p \in \operatorname{Int} D_{1}$. Let $D_{1}^{\prime}$ be a subdisk of $D_{1}$ such that $\pi\left(D_{1}^{\prime}\right) \subset \pi(B)$, and, for each $\xi>0$, let $X^{\xi}$ be the union of all vertical intervals of diameter no less than $\xi$ in $S \cup \operatorname{Int} S$ that intersect $D_{1}^{\prime}$. It is an exercise to see that $X^{\xi}$ is closed, and it follows from [6, Theorem 5] that $X^{\xi}$ is a *taming set. Now consider a point $q$ in $D_{1}^{\prime}$ but not in $X^{1 / i}$ for any $i$. It follows that $q$ lies in no vertical interval in $S \cup I n t S$. Thus $L(q)$ does not pierce $D_{1}^{\prime}$ at $q$, and $L(q)$ must pierce $D_{1}^{\prime}$ at some other point $t$ by the choice of $B$. Let $D$ be a disk in $D_{1}^{\prime}$ with $t$ in its interior such that $q \notin D$ and $L(q)$ links $\mathrm{Bd} D$. Then there is a disk $D_{q}$ in $D_{1}^{\prime}-D$ such that $q \in \operatorname{Int} D_{q}$ and each vertical line through $D_{q}$ links $\operatorname{Bd} D$. Thus such a line intersects both $D$ and $D_{2}$. This means that $D_{q}$ has vertical order 3 and is tame [7, Theorem 2.3]. Now we see that each point of $D_{1}^{\prime}$ either lies in the interior of a tame disk in $D_{1}^{\prime}$ or lies in $\bigcup_{1}^{\infty} X^{1 / i}$. Since a tame disk is a *-taming set and a countable number of tame disks suffice to cover $D_{1}^{\prime}-\bigcup_{1}^{\infty} X^{1 / i}$, we see that $D_{1}^{\prime}$ lies in a ${ }^{*}$-taming set of the form $\left(\bigcup_{1}^{\infty} X^{1 / i}\right) \cup$ (a countable collection of tame disks) in $S \cup \operatorname{Int} S$ [5, Theorem 3.7 and Corollary 3.8]. Thus $S$ is locally tame at $p$ from $E^{3}-(S \cup \operatorname{Int} S)$ by the definition of a ${ }^{*}$-taming set. Since $S$ is locally tame from Int $S$ [11], it follows that $S$ is locally tame at $p$.

Lemma 3.2. If $M$ is a continuum in $W(S)$ and $S$ is a 2-sphere having vertical order 5 , then $M$ is tame.

Proof. We may assume that $M$ is nondegenerate since singleton sets always lie on tame spheres. From the previous lemma it is clear that $\pi(M) \subset \mathrm{Bd} \pi(\operatorname{Int} S)$. Let $U=\operatorname{Int} S$ and let $X$ be the component of $\mathrm{Bd} \pi(U)$ containing $\pi(M)$. We shall show the existence of a space homeomorphism $H: E^{3} \rightarrow E^{3}$ such that $\pi(H(M))$ is either an arc or a simple closed curve. Then $H(M)$ is clearly tame since it lies in $\pi^{-1}(\pi(H(M)))$.

The continuum $X$ can be shown locally connected as in [7, Part 0.2]. Notice that $\pi(U)$ is open and connected. We let $U^{\prime}$ be the component of $E^{2}-X$ containing $\pi(U)$ and for convenience in what follows we assume that $U^{\prime}$ is bounded. Notice that $\operatorname{cl}\left(U^{\prime}\right)=X \cup U^{\prime}$ since every point of $S$ is accessible from Int $S$. Let $B^{2}=\left\{(x, y) \mid x^{2}+\right.$ $\left.y^{2} \leqq 1\right\} \subset E^{2}$. There is a continuous function $f: B^{2} \rightarrow \operatorname{cl}\left(U^{\prime}\right)$ such that 
$f \mid$ Int $B^{2}$ is a hemeomorphism of Int $B^{2}$ onto $U^{\prime}$ and $f^{-1}(x)$ is a totally disconnected subset of $S^{1}=\mathrm{Bd} B^{2}$ for each $x \in X$ (see [12, p. 186]). Now we follow [7, $\S \S 2.1,2.2,2.3$, and 2.4] to find a homeomorphism $H$ of $E^{3}$ onto $E^{3}$ such that $\pi\left(H\left(\pi^{-1}(X) \cap S\right)\right)$ is a simple closed curve. Thus $\pi(H(M))$ is either an arc or a simple closed curve since $\pi(H(M)) \subset$ $\pi\left(H\left(\pi^{-1}(X) \cap S\right)\right)$.

In the case where $U^{\prime}$ is not bounded the map $f$ above takes $E^{2}-$ Int $B^{2}$ onto $\mathrm{cl}\left(U^{\prime}\right)$ and causes some notational difficulties when we try to follow [7] as above. However, [7] still serves as an outline and we leave the details to the reader.

LEMma 3.3. If $M$ is a nondegenerate continuum in $W(S)$ and $S$ is a 2-sphere having vertical order 5 , then each point of $M$ is a limit point of $W(S)-M$.

Proof. Suppose some point $p \in M$ is not a limit point of $W(S)-M$, and choose a disk $D$ on $S$ such that $p \in \operatorname{Int} D, \mathrm{Bd} D$ is tame [3], and $D \cap W(S) \subset M$. Let $X=M \cup(\mathrm{Bd} D)$, and let $S^{\prime}$ be a 2 -sphere containing $M \cup D$ that is locally tame modulo $X[1]$. From Lemma 3.2 we see that $X$ is a taming set [4, Theorem 8.1.6, p. 320]. Thus $S^{\prime}$ is tame. This is a contradiction and the result follows.

Lemma 3.4. If $D$ is a disk in a 2-sphere $S$, $S$ has vertical order 5, $p \in \operatorname{Int} D$, and $V$ is an open subset of $E^{3}$ such that $p \in V$ and, for each vertical line $L$ piercing $D$ at a point in $V, L \cap$ Int $S$ has exactly one component whose closure intersects $D$, then $D$ is locally tame at $p$.

Proof. If $L(p)$ intersects Int $S$, then the conclusion of Lemma 3.4 follows from Lemma 3.1. Thus we now assume $L(p) \cap \operatorname{Int} S=\varnothing$. Choose a 2-sphere $H$ in the shape of a right circular cylinder such that $p \in \operatorname{Int} H, H \cap S \subset D, \operatorname{Bd} D \subset \operatorname{Ext} H,[L \cap(\operatorname{Int} H)] \cap S=\{p\}$, the top and bottom disks $T$ and $D$ of $H$ lie in Ext $S$, and each vertical line intersecting $H$ also intersects $V$.

Let $X$ be a component of $(\operatorname{Int} S) \cap H$, and let $K=\operatorname{Bd} X$. We shall show that $X \cup K$ is a disk by showing that $K$ is a simple closed curve. To show that $K$ is connected it suffices to prove that each simple closed curve $J$ in $X$ bounds a disk in $X$. Such a curve $J$ cannot be essential on the annulus $H-D \cup T$ since $J$ would link $L(p)$ while $L(p) \subset(\operatorname{Ext} S) \cup S$ and $J \subset \operatorname{Int} S$. Thus $J$ must bound a disk $E$ in $H-D \cup T$. From the hypothesis of Lemma 3.4 it is clear that $E \subset X$. Thus $K$ is connected. The fact that $K$ has vertical order 5 insures that $K$ is arcwise accessible from both its complementary domains in $H$, and this implies that $K$ is a simple closed curve.

Thus the closure of each component of (Int $S) \cap H$ is a spanning 
disk for the 3-cell $C=S \cup \operatorname{Int} S$. There can be at most a countable collection $\left\{D_{1}, D_{2}, \cdots\right\}$ of these spanning disks since their interiors are pairwise disjoint. The fact that $D$ has vertical order 5 insures that $\left\{D_{i}\right\}$ is a null sequence. We use these spanning disks to construct a 2-sphere $S^{\prime}$ containing $p$ and lying in $D \cup\left(\bigcup_{1}^{\infty} D_{i}\right)$ and in $H \cup \operatorname{Int} H$. From the hypothesis on $D$ we see that the interior of $S^{\prime}$ is vertically connected; thus $S^{\prime}$ is tame [7, Main Theorem]. This means that $D$ is locally tame at $p$.

THEOREM 3.5. If a 2-sphere $S$ in $E^{3}$ has vertical order 5, then $S \cup \operatorname{Int} S$ is a 3-cell and $S$ is locally tame from Ext $S$ modulo a 0-dimensional set.

Proof. That $C=S \cup \operatorname{Int} S$ is a 3-cell follows from [11]. It remains to show that the set $W$ of wild points of $S$ is 0 -dimensional. Suppose to the contrary that there is a nondegenerate continuum $M$ lying in $W$. Since $C$ is a 3-cell there is an embedding $g: M \times[0,1] \rightarrow C$ such that $G=g(M \times[0,1]) \subset \operatorname{Int} S$ and $g(m, 0)=m$ for every $m \in M$. We let $F=g(M \times[0,1])$, and we note that it follows from Lemma 3.1 that $\pi(M)$ lies in the boundary of $\pi(F)$ in $E^{2}$. For the same reason, $\pi(G) \cap \pi(M)=\varnothing$. Let $U$ be a disk in $E^{2}$ and let $p^{\prime}$ be a point of Int $U$ such that $U \cap(\pi(\operatorname{Bd} F)) \subset \pi(M)$ and $p^{\prime} \in \pi(M)$. Choose a point $p$ in $M \cap \pi^{-1}\left(p^{\prime}\right)$. In the next paragraph we show the existence of a disk $E$ in $S$ with $p \in \operatorname{Int} E$ and $\pi(E) \subset U \cap \pi(F)$.

The difficulty in choosing $E$ is the requirement that $\pi(E) \subset \pi(F)$. If no such $E$ exists there must exist a sequence $\left\{p_{i}\right\}$ of points of Int $S$ converging to $p$ such that $\pi\left(p_{i}\right) \in U-\pi(F)$ for each $i$. Using the 0 -ULC of Int $S$ it is easy to select a point $g \in G \subset \operatorname{Int} S$ close enough to $p$ and an integer $N$ large enough that $g$ and $p_{N}$ are the end points of an arc $A$ in Int $S$ where $\pi(A) \subset U$. Now $\pi(A)$ contains an are with one end point $a$ in $\pi(G)$ and the other end point $b$ in $U-\pi(F)$. If this arc is traversed from $b$ to $a$, then there is a first point $f$ of $\pi(F)$ encountered. This point $f$ clearly belongs to $\mathrm{Bd} \pi(F)$. This contradiction establishes the existence of $E$.

Now that the existence of $E$ is clear we proceed by using Lemma 3.3 to pick a point $q$ in $E \cap(W-M)$. Let $V$ be an open ball centered at $q$ such that $V \cap S \subset E$ and $V \cap F=\varnothing$. Since $L(q) \cap \operatorname{Int} S=\varnothing$ (see Lemma 3.1) there are open balls $B_{1}$ and $B_{2}$ centered at points above and below $q$, respectively, that lie in (Ext $S) \cap V$. We choose a disk $D$ in $V \cap S$ with $q \in \operatorname{Int} D$ vertically between $B_{1}$ and $B_{2}$ such that $\pi(D) \subset \pi\left(B_{1}\right) \cap \pi\left(B_{2}\right)$. We shall show that $D$ is locally tame at $q$ to obtain a contradiction to $q \in W$.

In order to apply Lemma 3.4 we must show that if a vertical line $L$ pierces $D$ at a point of $V$, then $L \cap \operatorname{Int} S$ has exactly one 
component whose closure intersects $D$. Suppose to the contrary that for some such line $L$ there are two components $X$ and $Y$ of $L \cap$ Int $S$ whose closures intersect $D$. Now $X \cup Y \subset V$ since $D$ lies between $B_{1}$ and $B_{2}$. Since $L \cap \operatorname{Int} S=\varnothing$ and $\pi(D) \subset \pi(F)$, we see that $L \cap G \neq \varnothing$. Thus $L \cap$ (Int $S$ ) has a third component $Z$, different from both $X$ and $Y$ because $Z$ lies either above $B_{1}$ or below $B_{2}$. Now the only way to avoid there being 6 points in $L \cap S$ is for $X$ and $Y$ to share an end point $x$. In this case there is a point $e$ of $\operatorname{Ext} S$ close enough to $x$ to insure that there are three components of $L(e) \cap \operatorname{Int} S$ with pairwise disjoint closures. Now $L(e) \cap S$ contains 6 points contrary to the hypothesis.

4. Examples and questions. One can use a countably infinite null sequence of Fox-Artin [8] "feelers" whose wild points form a dense subset of an arc to see that a vertically countable 2-sphere can have an arc in its wild set. Thus Corollary 2.2 cannot be improved in this direction.

EXAMPLE 4.1. A wild 2-sphere $S$ having vertical order 6 such that $W(S)$ is not 0 -dimensional. In Figure 1 we see an embedding of

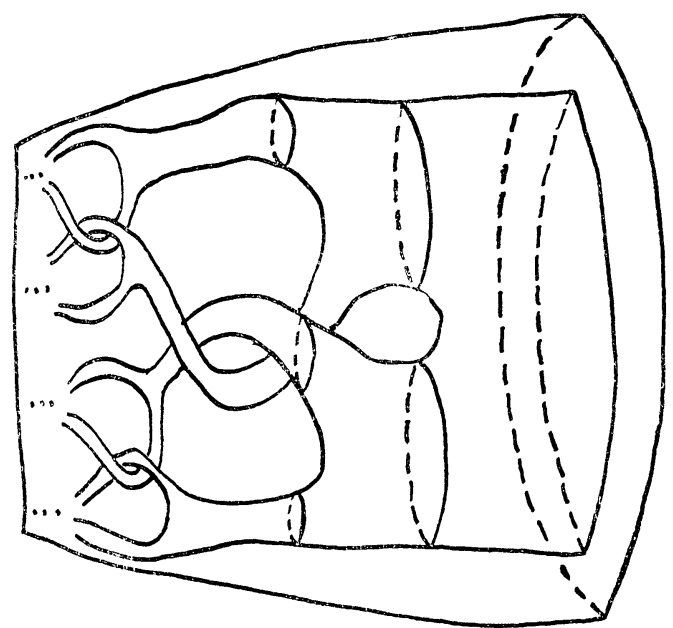

FIgURE 1.

the Alexander Horned Sphere, having vertical order 4, inside a wedgeshaped 3-cell in $E^{3}$. We attach a null sequence of such wedges to a right circular cone, as indicated in Figure 2, to obtain the desired example $S$. Notice that $W(S)$ is the union of a tame simple closed curve with countably infinite number of tame Cantor sets. Furthermore, every point of $S$ is a piercing point of $S$.

In Example 4.1 we see that every nondegenerate continuum in $W(S)$ is tame. 


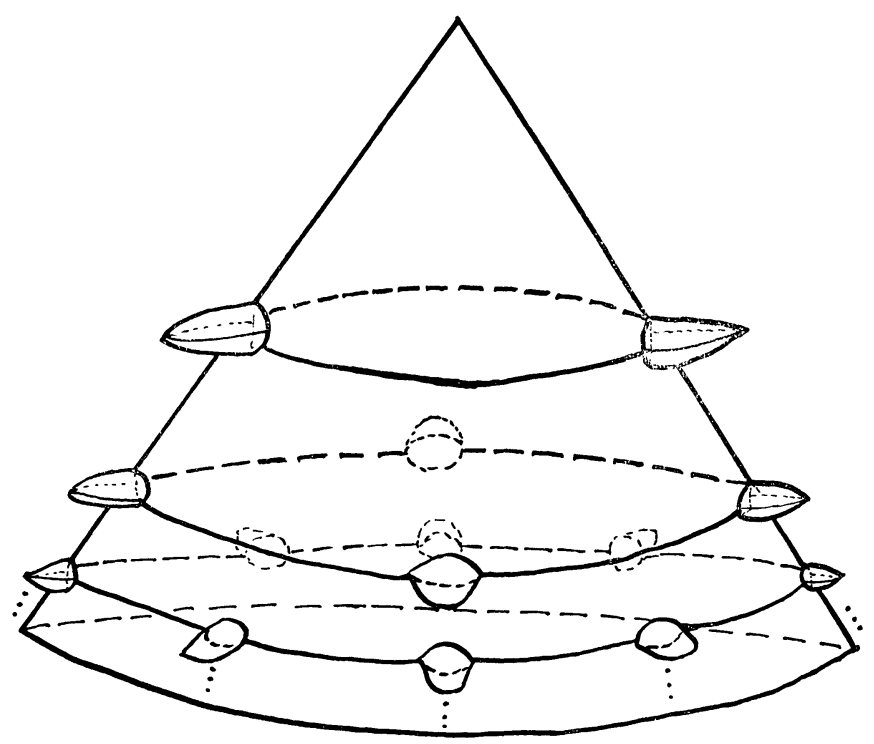

Figure 2.

Question 4.2. If $S$ is a 2-sphere in $E^{3}$ having finite vertical order, then must every nondegenerate continuum in $W(S)$ be tame?

We do not know the answer to Question 4.2 even when "vertical order $n$ " replaces "finite vertical order", unless $n \leqq 5$ where Theorem 3.5 applies. The proof of Lemma 3.2 shows an affirmative answer to Question 4.2 if it is also known that $\pi(W(S)) \cap \pi(\operatorname{Int} S)=\varnothing$.

\section{REFERENCES}

1. R. H. Bing, Approximating surfaces with polyhedral ones, Ann. of Math., (2) 65 (1957), 456-483.

2. Conditions under which a surface in $E^{3}$ is tame, Fund. Math., 47 (1959), 105-139.

3. - Each disk in $E^{3}$ contains a tame arc, Amer. J. Math., 84 (1962), 583-590.

4. C. E. Burgess and J. W. Cannon, Embeddings of surfaces in $E^{3}$, Rocky Mountain

J. Math., 1 (1971), 259-344.

5. J. W. Cannon, *-taming sets for crumpled cubes, I: Basic properties, Tran. Amer. Math. Soc., 161 (1971), 429-440.

6. - *-taming sets for crumpled cubes, II: Horizontal sections in closed sets, Trans. Amer. Math. Soc., 161 (1971), 441-446.

7. J. W. Cannon and L. D. Loveland, A 2-sphere in $E^{3}$ with vertically connected interior is tame, Trans. Amer. Math. Soc., (to appear).

8. R. H. Fox and E. Artin, Some wild cells and spheres in three-dimensional space, Ann. of Math., (2) $\mathbf{4 9}$ (1948), 979-990.

9. W. Hurewicz and H. Wallman, Dimension Theory, Princeton Math. Series, Vol. 4, Princeton Univ. Press, Princeton, N. J., 1941.

10. R. A. Jensen and L. D. Loveland, Surfaces of vertical order 3 are tame, Bull. Amer. Math. Soc., 76 (1970), 151-154.

11. L. D. Loveland, A 2-sphere of vertical order 5 bounds a 3-cell, Proc. Amer. Math.

Soc., 26 (1970), 674-678. 
12. M. H. A. Newman, Elements of the Topology of Plane Sets of Points, First Edition, Cambridge Univ. Press, Cambridge, 1937.

Received November 21, 1972.

UTAH State UNIVERSITY 



\section{PACIFIC JOURNAL OF MATHEMATICS}

\section{EDITORS}

RICHARD ARENS (Managing Editor)

University of California

Los Angeles, California 90024

\section{R. A. Beaumont \\ University of Washington \\ Seattle, Washington 98105}

\section{J. DugundjI*}

Department of Mathematics University of Southern California Los Angeles, California 90007

D. Gilbarg and J. Milgram

Stanford University

Stanford, California 94305

\section{ASSOCIATE EDITORS}
E. F. BeCKenBaCH
B. H. NeumanN
F. WOLF
K. YoSHIDA

\section{SUPPORTING INSTITUTIONS}

\author{
UNIVERSITY OF BRITISH COLUMBIA \\ CALIFORNIA INSTITUTE OF TECHNOLOGY \\ UNIVERSITY OF CALIFORNIA \\ MONTANA STATE UNIVERSITY \\ UNIVERSITY OF NEVADA \\ NEW MEXICO STATE UNIVERSITY \\ OREGON STATE UNIVERSITY \\ UNIVERSITY OF OREGON \\ OSAKA UNIVERSITY
}

\author{
UNIVERSITY OF SOUTHERN CALIFORNIA \\ STANFORD UNIVERSITY \\ UNIVERSITY OF TOKYO \\ UNIVERSITY OF UTAH \\ WASHINGTON STATE UNIVERSITY \\ UNIVERSITY OF WASHINGTON

$* * *$
$*$
AMERICAN MATHEMATICAL SOCIETY
NAVAL WEAPONS CENTER

The Supporting Institutions listed above contribute to the cost of publication of this Journal, but they are not owners or publishers and have no responsibility for its content or policies.

Mathematical papers intended for publication in the Pacific Journal of Mathematics should be in typed form or offset-reproduced, (not dittoed), double spaced with large margins. Underline Greek letters in red, German in green, and script in blue. The first paragraph or two must be capable of being used separately as a synopsis of the entire paper. Items of the bibliography should not be cited there unless absolutely necessary, in which case they must be identified by author and Journal, rather than by item number. Manuscripts, in duplicate if possible, may be sent to any one of the four editors. Please classify according to the scheme of Math. Rev. Index to Vol. 39. All other communications to the editors should be addressed to the managing editor, or Elaine Barth, University of California, Los Angeles, California, 90024.

100 reprints are provided free for each article, only if page charges have been substantially paid. Additional copies may be obtained at cost in multiples of 50 .

The Pacific Journal of Mathematics is issued monthly as of January 1966. Regular subscription rate: $\$ 60.00$ a year (6 Vols., 12 issues). Special rate: $\$ 30.00$ a year to individual members of supporting institutions.

Subscriptions, orders for back numbers, and changes of address should be sent to Pacific Journal of Mathematics, 103 Highland Boulevard, Berkeley, California, 94708.

PUBLISHED BY PACIFIC JOURNAL OF MATHEMATICS, A NON-PROFIT CORPORATION

Printed at Kokusai Bunken Insatsusha (International Academic Printing Co., Ltd.), 270, 3-chome Totsuka-cho, Shinjuku-ku, Tokyo 160, Japan

* C. R. DePrima California Institute of Technology, Pasadena, CA 91109, will replace J. Dugundji until August 1974. 


\section{Pacific Journal of Mathematics}

Vol. 50, No. $2 \quad$ October, 1974

Mustafa Agah Akcoglu, John Philip Huneke and Hermann Rost, A counter example to the Blum Hanson theorem in general spaces .............

Huzihiro Araki, Some properties of modular conjugation operator of von

Neumann algebras and a non-commutative Radon-Nikodym theorem

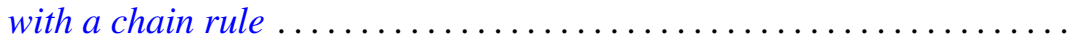

E. F. Beckenbach, Fook H. Eng and Richard Edward Tafel, Global properties of rational and logarithmico-rational minimal surfaces .....

David W. Boyd, A new class of infinite sphere packings ............. 383

K. G. Choo, Whitehead Groups of twisted free associative algebras ........

Charles Kam-Tai Chui and Milton N. Parnes, Limit sets of power series

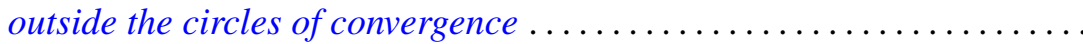

Allan Clark and John Harwood Ewing, The realization of polynomial

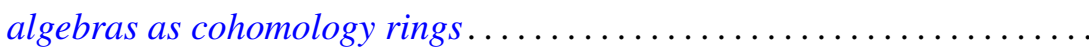

Dennis Garbanati, Classes of circulants over the p-adic and rational

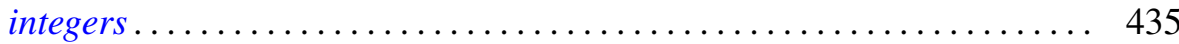

Arjun K. Gupta, On a "square" functional equation ................... 449

David James Hallenbeck and Thomas Harold MacGregor, Subordination and extreme-point theory ............................. 455

Douglas Harris, The local compactness of $v X \ldots \ldots . . . . . . . . . . . .4469$

William Emery Haver, Monotone mappings of a two-disk onto itself which fix the disk's boundary can be canonically approximated by homeomorphisms .................................. 477

Norman Peter Herzberg, On a problem of Hurwitz .................. 485

Chin-Shui Hsu, A class of Abelian groups closed under direct limits and subgroups formation ............................... 495

Bjarni Jónsson and Thomas Paul Whaley, Congruence relations and multiplicity types of algebras.....................

Lowell Duane Loveland, Vertically countable spheres and their wild sets.

Nimrod Megiddo, Kernels of compound games with simple components ....

Russell L. Merris, An identity for matrix functions ........ . .

E. O. Milton, Fourier transforms of odd and even tempered distributions ...

Dix Hayes Pettey, One-one-mappings onto locally connected generalized continua

Mark Bernard Ramras, Orders with finite global dimension

Doron Ravdin, Various types of local homogeneity. .

George Michael Reed, On metrizability of complete Moore spaces ...

Charles Small, Normal bases for quadratic extensions ..

Philip C. Tonne, Polynomials and Hausdorff matrices.... . . 